\title{
Enzyme-substrate complexes of the quinate/ shikimate dehydrogenase from Corynebacterium glutamicum enable new insights in substrate and cofactor binding, specificity, and discrimination
}

\begin{abstract}
Quinate dehydrogenase (QDH) catalyzes the reversible oxidation of quinate to 3-dehydroquinate by nicotineamide adenine dinucleotide (NADH) and is involved in the catabolic quinate metabolism required for the degradation of lignin. The enzyme is a member of the family of shikimate/quinate dehydrogenases (SDH/QDH) occurring in bacteria and plants. We characterized the dual-substrate quinate/shikimate dehydrogenase (QSDH) from Corynebacterium glutamicum (CglQSDH) kinetically and revealed a clear substrate preference of $\mathrm{CglQSDH}$ for quinate compared with shikimate both at the $\mathrm{pH}$ optimum and in a physiological $\mathrm{pH}$ range, which is a remarkable contrast to closely related SDH/QDH enzymes. With respect to the cosubstrate, $\mathrm{CglQSDH}$ is strictly $\mathrm{NAD}(\mathrm{H})$ dependent. These substrate and cosubstrate profiles correlate well with the details of three atomic resolution crystal structures of $\mathrm{CglQSDH}$ in different functional states we report here: with bound $\mathrm{NAD}^{+}$(binary complex) and as ternary complexes with NADH plus either shikimate or quinate. The CglQSDH-NADH-quinate structure is the first complex structure of any member of the SDH/QDH family with quinate. Based on this novel structural information and systematic sequence and structure comparisons with closely related enzymes, we can explain the strict NAD $(\mathrm{H})$ dependency of CglQSDH as well as its discrimination between shikimate and quinate.
\end{abstract}

Keywords: binary complex structure; enzyme kinetics; mechanism of catalysis; shikimate/quinate dehydrogenase family; substrate and cofactor binding; ternary complex structure.

\footnotetext{
*Corresponding authors: Astrid Höppner, Institute for Biochemistry, University of Cologne, Otto-Fischer Straße 12-14, D-50674 Cologne, Germany; and Crystal Farm and X-Ray Facility, Heinrich Heine University Düsseldorf, Universitätsstraße 1, D-40225 Düsseldorf, Germany, e-mail: astrid.hoeppner@hhu.de; and Karsten Niefind, Institute for Biochemistry, University of Cologne, Otto-Fischer Straße 12-14, D-50674 Cologne, Germany, e-mail: karsten.niefind@uni-koeln.de
}

Dietmar Schomburg: Institute for Biochemistry, University of Cologne, Otto-Fischer Straße 12-14, D-50674 Cologne, Germany; and Department of Bioinformatics and Biochemistry, Institute for Biochemistry and Biotechnology, Technische Universität Braunschweig, Langer Kamp 19B, D-38106 Braunschweig, Germany

\section{Introduction}

The enzyme quinate dehydrogenase $(\mathrm{QDH})$ catalyzes the reversible conversion of quinate to 3-dehydroquinate and is involved in the catabolic quinate pathway. The first $\mathrm{NAD}(\mathrm{H})$-dependent QDH was reported in 1954 from Aerobacter aerogenes (Mitsuhashi and Davis, 1954). Meanwhile, the QDHs are assigned to the family of bacterial shikimate/quinate dehydrogenases (SDH/QDH), of which a number of functional classes were specified to date. Enzymologically, all of them belong either to the EC class 1.1.1.24 (QDHs) or to EC 1.1.1.25 (SDHs) (Schomburg et al., 2013).

The first SDH/QDH class, a strictly nicotineamide adenine dinucleotide phosphate $[\mathrm{NADP}(\mathrm{H})]$-dependent SDH, was discovered in Escherichia coli (Yaniv and Gilvarg, 1955; Chaudhuri and Coggins, 1985) and was referred to as AroE. Physiologically, AroE is localized in the anabolic shikimate pathway. The second class are the YdiBs (Benach et al., 2003), which are characterized as dual-substrate $\mathrm{SDH} / \mathrm{QDH}$-converting (dehydro-)quinate or (dehydro-)SDH and using $\mathrm{NAD}(\mathrm{H})$ as well as $\mathrm{NADP}(\mathrm{H})$ as a cosubstrate. In 2008, Singh et al. designated a novel $\mathrm{SDH} / \mathrm{QDH}$ subclass (YdiB2) that is distinct from the previously described $E$. coli YdiB (Singh et al., 2008). The enzymes of the third class were paraphrased as 'shikimate dehydrogenase-like proteins' (SDH-L) (Singh et al., 2005); SDH-Ls were first identified in Haemophilus influenzae and showed catalytic efficiencies several magnitudes lower than AroE. A fourth functionally distinct class of the SDH/QDHs was identified by Singh et al. (2008) and named AroE-like 1 (Ael1). In addition to these four 'classical' SDH/QDH subfamilies, a fifth class, denoted as 'Rifl', was recently defined on the basis of biochemical 
and structural characteristics (Peek et al., 2013). Compared with AroE, the RifI2 enzyme exhibits lower activity with shikimate as a substrate and a strong preference for $\mathrm{NAD}^{+}$instead of $\mathrm{NADP}^{+}$as a cofactor. Thus, an increasing diversity of the SDH/QDH enzyme family has been revealed within the last decade, but - as we will show here - the enzyme that we used for this study and that naturally occurs in Corynebacterium glutamicum encoded by the $q s u D$ gene (Teramoto et al., 2009) does not belong to any of the described classes. Because of its particular enzymological properties, we refer to this enzyme here as 'quinate/shikimate dehydrogenase' (QSDH).

Quinate metabolism is connected to the much better understood shikimate pathway, which for its part consists of seven enzymatic steps starting with the condensation of phosphoenolpyruvate and erythrose-4-phosphate and ends with the formation of chorismate (Herrmann and Weaver, 1999). The shikimate pathway is involved in the biosynthesis of aromatic amino acids, folates, vitamins, quinones, and a diversity of other aromatic compounds in bacteria, plants, fungi, and apicomplexan parasites, but not in animals (Roberts et al., 1998; Herrmann and Weaver, 1999). Some of the aromatic metabolites are essential for the survival of these organisms. The combination of these facts renders the shikimate pathway an attractive target for the design of antimicrobial and herbicidal agents (Davies et al., 1994; Coggins et al., 2003).

Intermediates of the shikimate pathway represent two key precursors of the quinate pathway, namely 3-dehydroquinate and 3-dehydroshikimate (Figure 1). This quinate pathway is composed of three enzymatic reactions: (1) the $\mathrm{NAD}^{+}$-dependent oxidation of quinate to 3-dehydroquinate, (2) the dehydration to 3-dehydroshikimate, and (3) the conversion to protocatechuate (Figure 1). These reactions are catalyzed by the enzymes QDH, 3-dehydroquinate dehydratase, and 3-dehydroshikimate dehydratase, respectively (Herrmann, 1995). Finally, protocatechuate is degraded to succinate and acetyl-CoA via the $\beta$-ketoadipate pathway (Grund and Kutzner, 1998). Alternatively, 3-dehydroquinate and 3-dehydroshikimate can be converted via quinate to chlorogenate, a widespread metabolite that protects plants against fungal attack and UV radiation.

The quinate pathway has been well characterized in several bacteria and fungi (Reinert et al., 1981; Charles et al., 1985; Elsemore and Ornston, 1994), where it is of particular importance for the degradation of aromatic compounds. These occur due to environmental pollution by toxic aromatic hydrocarbons and also in the context of the degradation of lignin, which represents a quarter of the terrestrial biomass. Thus, via the recovery of lignin

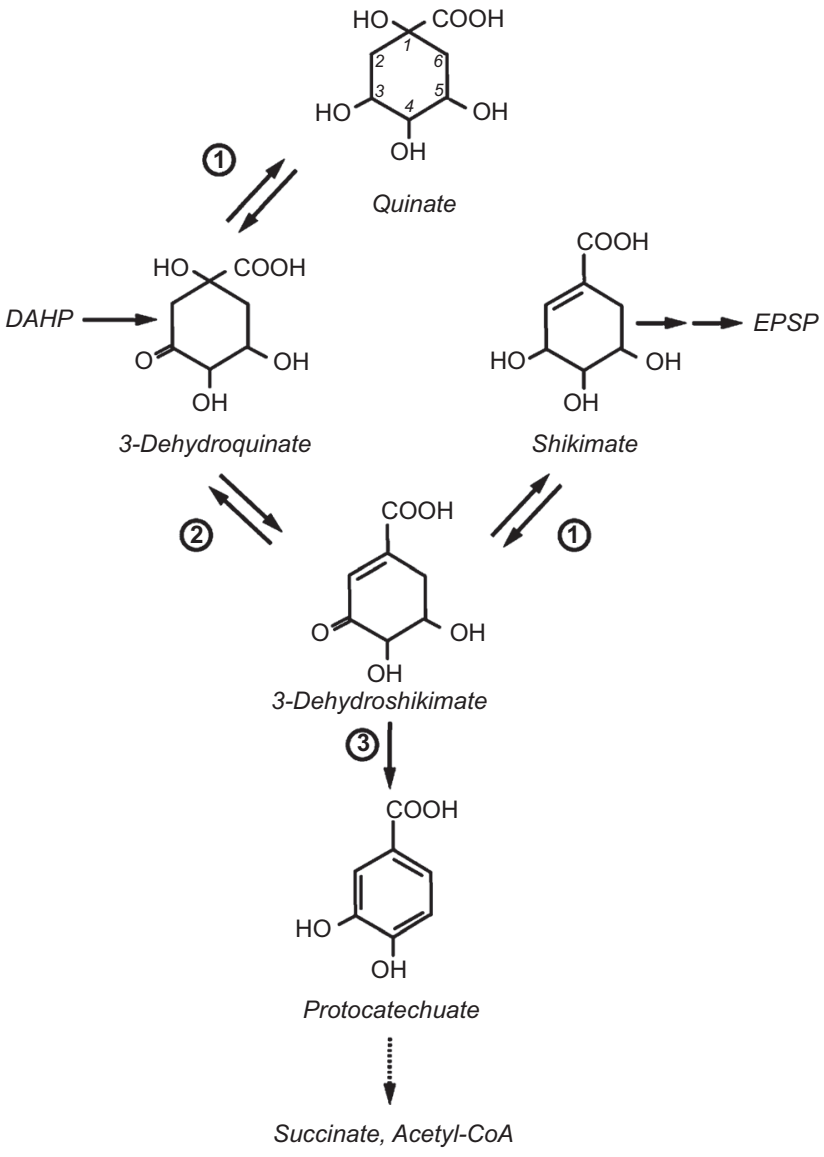

Figure 1 Schematic overview of the quinate pathway. DAHP=3-deoxy-D-arabino-heptulosonate-7-phosphate; $\mathrm{EPSP}=5$-enolpyruvyl-shikimate-3-phosphate; 1 =quinate (shikimate) dehydrogenase; $2=3$-dehydroquinate dehydratase; 3=3-dehydroshikimate dehydratase (according to Herrmann, 1995).

and other aromatic plant material, QDH and the quinate pathway play a decisive role in the global carbon cycle (Harwood and Parales, 1996). This is especially true for QSDH from C. glutamicum (CglQSDH) because the latter is a soil bacterium, however, with a most remarkable career for bulk production of amino acids in biotechnology (Ikeda and Katsumata, 1992; Sahm et al., 2000).

Considerable efforts have been made to characterize shikimate- and quinate-related enzymes on a threedimensional (3D) level and rationalize the structural principles underlying the details of their enzymatic profiles. In fact, for all enzymes of the shikimate pathway, structural information was published (Stallings et al., 1991; Carpenter et al., 1998; Gourley et al., 1999; Shumilin et al., 1999; Roszak et al., 2002; Maclean and Ali, 2003) as well as for CglQSDH, PDB-ID 2NLO (Schoepe et al., 2008). However, although only an apo-structure is available for the latter, the aforementioned homologues from the AroE, 
YdiB, SDH-L, Ael1, and Rifl classes were extensively structurally characterized both as apo-enzymes and as binary complexes, but often as well as ternary complexes using various natural sources such as E. coli (Michel et al., 2003), Methanococcus jannaschii (Padyana and Burley, 2003), H. influenzae (Ye et al., 2003), Aquifex aeolicus (Gan et al., 2007), Thermus thermophilus (Bagautdinov and Kunishima, 2007), Staphylococcus epidermis (Han et al., 2009), and Pseudomonas putida (Peek et al., 2011). Even a ternary complex of the bifunctional $\mathrm{SDH} /$ dehydroquinate dehydratase from Arabidopsis thaliana (Singh and Christendat, 2006, 2007) was published; the structure of the closely related YdiB enzyme was solved in 2003 (Benach et al., 2003; Michel et al., 2003). Yet, despite all efforts, a functional complex with quinate was never crystallized with any of these enzymes so far.

Based on all these structures, it was established that all members of the SDH/QDH family possess a similar 3D fold, despite their relatively low amino acid sequence identity. Their root mean square deviation (RMSD) values compared with the structure of the $\mathrm{CglQSDH}$ range from 1.55 to $2.58 \AA$ over a length of 224-262 amino acids (Table S2 in the online supplement). They all show a typical two-domain fold consisting of an N-terminal catalytic or substrate-binding domain and a C-terminal or nucleotidebinding domain. The substrate-binding domain forms an open $\alpha / \beta$ sandwich with a central, mainly parallel six-stranded $\beta$ sheet flanked by four to five $\alpha$ helices. The nucleotide-binding domain exhibits a central six-stranded parallel $\beta$ sheet surrounded by four to six $\alpha$ helices. The latter domain contains a classical Rossmann fold.

Although various data of complexes are disposable, some questions remain unclear. The mechanism of catalysis is not completely understood in detail. In agreement, two highly conserved active site residues, a lysine and an aspartate, were assigned an important role during catalysis (Lindner et al., 2005; Singh and Christendat, 2006; Bagautdinov and Kunishima, 2007; Gan et al., 2007; Rodrigues et al., 2009; Peek et al., 2011). Under debate is also a conserved tyrosine, which may stabilize the catalytic intermediate (Han et al., 2009), or a putative conserved water molecule in the active site, which might mediate the proton transfer to the bulk solvent (Bagautdinov and Kunishima, 2007; Gan et al., 2007).

The main focus of the work presented here was to rationalize CglQSDH's substrate and cofactor specificity, i.e., to understand the way the enzyme discriminates between shikimate and quinate on the one hand and between NADH and NADPH on the other. Then, this knowledge should be applied to discover sequence- and/ or structure-based patterns within the SDH/QDH family members, which may govern the remarkable specificity profiles ranging from clear preferences to only one substrate/cofactor to the more or less equal usage of two chemically related compounds.

Here, we present the results of our biochemical and sequence-based analyses as well as three crystal structures of $\mathrm{CglQSDH}$ at atomic resolution, namely the binary complex with $\mathrm{NAD}^{+}$and two different ternary complexes both with NADH and with either quinate or shikimate.

\section{Results}

\section{Kinetic properties of QSDH}

Kinetic analyses of the CglQSDH were performed for the substrates quinate and shikimate as well as for the cofactors $\mathrm{NAD}^{+}$and $\mathrm{NADP}^{+}$. To obtain a comprehensive view, we determined the kinetic properties of the enzyme at two different $\mathrm{pH}$ values: at $\mathrm{pH}$ where the activity was highest (pH optimum: 9.0/9.5 for quinate and 10.0/10.5 for shikimate) and at physiological $\mathrm{pH}$ (7.5). The kinetic parameters are listed in Table 1.

At $\mathrm{pH}$ optimum, the $K_{\mathrm{M}}$ values for quinate and shikimate are 2.37 and $53.88 \mathrm{~mm}$, respectively. Despite of the two times higher turnover rate for shikimate $\left(k_{\text {cat }}=214.09 / \mathrm{s}\right)$, the catalytic efficiency for quinate is about 10 -fold higher $\left[k_{\text {cat }} / K_{\mathrm{m}}=44.05 /(\mathrm{s} \mathrm{mM})\right]$. The kinetic parameters for the cofactor $\mathrm{NAD}^{+}$are similar: the $K_{\mathrm{m}}$ value for $\mathrm{NAD}^{+}$is 0.28 and $0.46 \mathrm{~mm}$ with quinate and shikimate as substrate, respectively. The rates of turnover are also in the same range (223.06 and 205.78/s with quinate and shikimate, respectively).

At $\mathrm{pH}$ 7.5, a more physiological condition, these different catalytic properties become more obvious. The $K_{\mathrm{m}}$ values for quinate and shikimate decrease to 1.56 or $10.16 \mathrm{~mm}$ and the turnover rates decrease 2- and 7-fold, which result in approximately 10 -fold lower catalytic efficiency $\left[k_{\text {cat }} / K_{\mathrm{m}}=33.30 /(\mathrm{s} \mathrm{mM})\right.$ for quinate; $k_{\text {cat }} / K_{\mathrm{m}}=3.10 /$ (s $\mathrm{mm}$ ) for shikimate]. In summary, all results of the kinetic analysis indicate quinate as the main substrate of CglQSDH.

Kinetic measurements with $\mathrm{NADP}^{+}$applied rather than $\mathrm{NAD}^{+}$at $\mathrm{pH} 7.5$ or $\mathrm{pH}$ optimum revealed no conversion at all, regardless of which condition we chose (data not shown). Obviously - and in full coincidence with a recent report by Kubota et al. (2013) - $\mathrm{NADP}^{+}$cannot be converted or even bound by CglQSDH for the oxidation of either quinate or shikimate. Therefore, CglQSDH is a strictly $\mathrm{NAD}(\mathrm{H})$-dependent $\mathrm{QDH}$. Its kinetic parameters are in the same order of magnitude as those of other QDHs 
Table 1 Apparent kinetic parameters of $\mathrm{Cg}$ lQSDH under different $\mathrm{pH}$ conditions.

\begin{tabular}{lrrr}
\hline & $\boldsymbol{K}_{\mathrm{M}}(\mathrm{mM})$ & $\boldsymbol{k}_{\text {cat }}(\mathbf{1} / \mathbf{s})$ & $\boldsymbol{k}_{\text {cat }} / \boldsymbol{K}_{\mathrm{M}}[\mathbf{1} /(\mathbf{s ~ m M})]$ \\
\hline Optimum pH $^{\mathrm{a}}$ & & & \\
Quinate $^{\mathrm{b}}$ & $2.37 \pm 0.77$ & $104.87 \pm 43.78$ & $44.05 \pm 10.84$ \\
$\mathrm{NAD}^{+}($quinate) & $0.28 \pm 0.07$ & $223.06 \pm 27.04$ & $826.61 \pm 226.52$ \\
Shikimate $^{\mathrm{b}}$ & $53.88 \pm 15.43$ & $214.09 \pm 30.37$ & $4.24 \pm 1.38$ \\
$\mathrm{NAD}^{+}\left(\right.$shikimate) $^{\mathrm{d}}$ & $0.46 \pm 0.09$ & $205.78 \pm 29.58$ & $464.65 \pm 84.15$ \\
$\mathrm{pH} \mathrm{7.5}$ & & & \\
Quinate $^{\mathrm{b}}$ & $1.56 \pm 0.50$ & $50.82 \pm 17.31$ & $33.30 \pm 7.44$ \\
$\mathrm{NAD}^{+}$(quinate) $^{\mathrm{c}}$ & $0.13 \pm 0.03$ & $43.67 \pm 8.64$ & $329.14 \pm 20.52$ \\
Shikimate $^{\mathrm{b}}$ & $10.16 \pm 3.53$ & $30.13 \pm 7.84$ & $3.10 \pm 0.76$ \\
NAD $^{+}\left(\right.$shikimate) $^{\mathrm{d}}$ & $0.87 \pm 0.25$ & $61.12 \pm 13.77$ & $71.58 \pm 12.16$ \\
\hline
\end{tabular}

${ }^{a} \mathrm{pH}$ 9.0/9.5 for quinate and $\mathrm{pH}$ 10.0/10.5 for shikimate.

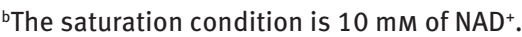

'The saturation condition is $16 \mathrm{~mm}$ quinate.

${ }^{\mathrm{d}}$ The saturation condition is $60 \mathrm{~mm}$ shikimate.

described previously (Mitsuhashi and Davis, 1954; Cain, 1972; Whiting and Coggins, 1974; Bruce and Cain, 1990; Kang and Scheibe, 1993; Michel et al., 2003; Lindner et al., 2005).

The overall kinetic profile of $\mathrm{CglQSDH}$ is comparable only to QDH from Lactobacillus plantarum (Whiting and Coggins, 1974) and YdiB2 from P. putida (Singh et al., 2008).

\section{Structure determination}

To rationalize the enzymological data obtained before, we determined the crystal structures of three functional complexes of CglQSDH (Table S1 in the online supplement): the binary complex with $\mathrm{NAD}^{+}$as well as the ternary complexes with NADH plus either shikimate or quinate.

All final models consist of 282 residues and 1 NAD(H) molecule. QSDH-NAD contains 441 water molecules, QSDH-quinate-NADH 369 waters molecules, and QSDHshikimate-NADH 388 water molecules, respectively. The ternary complexes additionally contain one substrate molecule (quinate or shikimate). Residues -19 up to 1, consisting of the N-terminal his ${ }_{6}$-tag, the AcTEV cleavage site, spacer amino acids, and the first amino acid of the native protein are disordered in all three cases, as judged by the lack of electron density for this region.

\section{Structure overview of $C$. glutamicum QSDH}

The protein parts of the three complex structures reported here and of the apo-CglQSDH published previously (Schoepe et al., 2008) are largely identical; they possess the two-domain architecture typical for members of the
SDH/QDH protein family consisting of an N-terminal catalytic or substrate-binding domain and a C-terminal or nucleotide-binding domain.

The N-terminal or catalytic domain comprises residues 1 to 113 and 256 to 283, whereas the C-terminal or nucleotide-binding domain is build up of residues 114 to 255 . The catalytic domain forms an open $\alpha / \beta$ sandwich, which is characteristic for enzymes of the SDH/QDH family (Michel et al., 2003; Ye et al., 2003). The domain consists of a six-stranded, mainly parallel $\beta$ sheet (strand order $\beta 2, \beta 1, \beta 3, \beta 5, \beta 6$, and $\beta 4$, where $\beta 5$ is antiparallel). This $\beta$ sheet is flanked by helices $\alpha 1$ and $\alpha 9$ at one side and $\alpha 2$, one $3_{10}$-helix, and $\alpha 3$ at the other (Figure 2, highlighted in blue). The C-terminal domain contains a six-stranded parallel $\beta$ sheet (strand order $\beta 9, \beta 8, \beta 7, \beta 10, \beta 11$, and $\beta 12$ ) sandwiched by three helices $(\alpha 6, \alpha 5, \alpha 4)$ on one face and by helices $\alpha 7, \alpha 8$, and a $3_{10}$-helix on the other (Figure 2, highlighted in green). The nucleotide-binding domain exhibits a classical Rossmann fold (Rossmann et al., 1974) with the sequence motif GxGGxG within the glycine-rich loop. Both domains are linked together by helices $\alpha 4$ and $\alpha 9$. The arrangement of these two domains creates a deep groove, in which the cofactor and substrate are located, and forms the active site. The CglQSDH appears to exist as dimer in solution as we reported previously (Schoepe et al., 2006, 2008).

\section{Description and analysis of QSDH active site}

\section{Cofactor-binding site}

The electron densities for the $\mathrm{NAD}(\mathrm{H})$ molecules were of high quality and allowed us to assign the positions of 
A
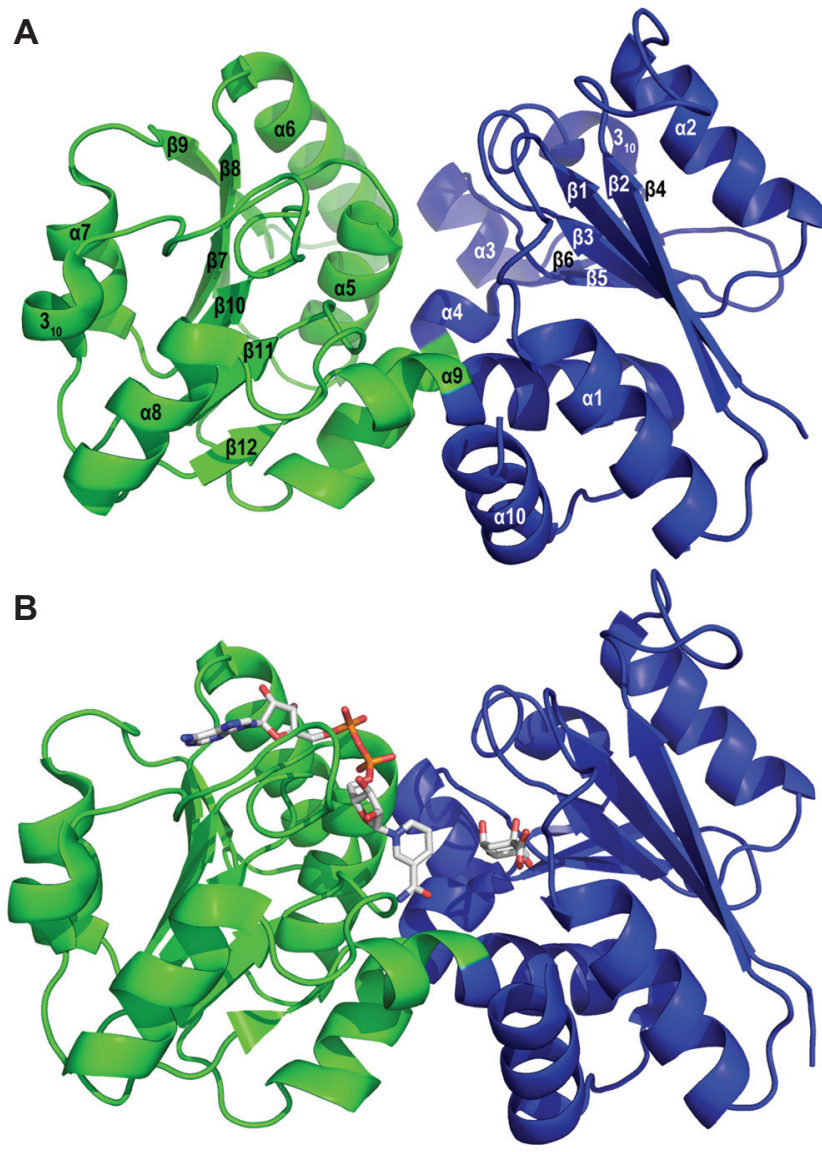

Figure 2 Cartoon diagram representing the polypeptide fold of CglQSDH.

Apo-QSDH with secondary structure assignments (A) and ternary complex of QSDH with NADH and quinate, shown as stick models (B). The catalytic domain is depicted in blue and the nucleotidebinding domain in green.

these ligand atoms unambiguously (Figure 3). In the presence of $\mathrm{NAD}^{+}, \mathrm{CglQSDH}$ crystallized in the same space group with similar unit cell dimensions, but under different crystallization conditions compared with the apoenzyme (Schoepe et al., 2008). Globally, we found that the catalytic domain moved away from the nucleotide-binding domain by $1.2 \AA$ after cofactor binding, thus enlarging the interdomain cavity. Specifically, the loop (Gly136-Val138) moved out of the cavity to release space for the $\mathrm{NAD}(\mathrm{H})$ molecule. Cofactor binding occurs in an extended groove in between the $\mathrm{N}$ - and $\mathrm{C}$-terminal domain, whereas most of the molecular interactions result from the C-terminal domain. The adenine part of the adenosine moiety forms hydrogen bonds only to some water molecules, whereas the ribose is bound by the side chains of Asp158 and Arg163. The diphosphate moiety contacts the glycine-rich loop (sequence motif GxGGxG comprising residues Gly134 to Gly139) and forms hydrogen bonds to Arg163 and the backbone nitrogen atom of Val138. The following ribose moiety interacts only with water molecules, whereas the nicotinamide moiety is cramped by the backbone nitrogen of Ala255 and backbone oxygen of Val228 and Gly251, respectively.

\section{Substrate-binding site}

We examined the substrate-binding site of $\mathrm{CglQSDH}$ analyzing the two different ternary complexes QSDH-quinateNADH and QSDH-shikimate-NADH in comparison to the binary $\mathrm{CglQSDH}-\mathrm{NAD}^{+}$complex. The substrate-binding site is located in the N-terminal domain, close to the nicotinamide ring of the cofactor (Figure 3).

Upon quinate binding, we observed a slight closure of the $\mathrm{N}$ - and C-terminal domain of $\mathrm{CglQSDH}$ so that the crevice becomes closer about $0.5 \AA$. The substrate quinate is anchored by numerous key interactions with the following residues: the carbonyl group of quinate is bound by the hydroxy groups of Ser17 and Thr19; the hydroxy groups of the $\mathrm{C} 1$ and $\mathrm{C} 3$ atom of the substrate form hydrogen bonds to side chain of Thr69, whereas the nitrogen atom of Lys73 binds to the hydroxy groups attached to $\mathrm{C} 3$ and $\mathrm{C} 4$, with the latter further interacting with the side chains of Asn94 and Asp110; the fourth hydroxy group at C5 forms hydrogen bonds to the amide group of Asp110 and the oxygen atom of the Gln258 side chain. A total of 11 hydrogen bonds cause a forcipate anchorage of the substrate molecule (Figure 4A). In comparison to the apoenzyme of $C g l \mathrm{QSDH}$, it is noteworthy that the side chain of Lys73 exhibits a sprawled conformation after quinate binding, which is required for interaction with the $\mathrm{C} 3$ and C4 hydroxy groups of the substrate molecule.

For the hydride ion transfer from $\mathrm{C} 3$ of quinate to $\mathrm{C} 4$ of $\mathrm{NAD}^{+}$, a particular distance between these atoms is of particular importance. In the crystal structure, the nicotinamide ring is located in a suitable orientation for the $\mathrm{H}^{-}$transfer. Nevertheless, with $4.27 \AA$, the distance between the two reactive centers is still too large, so that further domain closure enabling finally a fully functional complex can be anticipated.

In case of shikimate binding, we found a somewhat different situation. In principle, the above-mentioned residues except Thr19 are involved in shikimate binding (Figure 4B), but only seven polar interactions are achieved, three of which are weaker pronounced (see Table S3 in the online supplement). Remarkable is the appearance of an alternative side-chain conformation of Lys73. One part of the Lys73 side chains in the crystal exhibits the sprawled conformation as found upon quinate binding, the other 


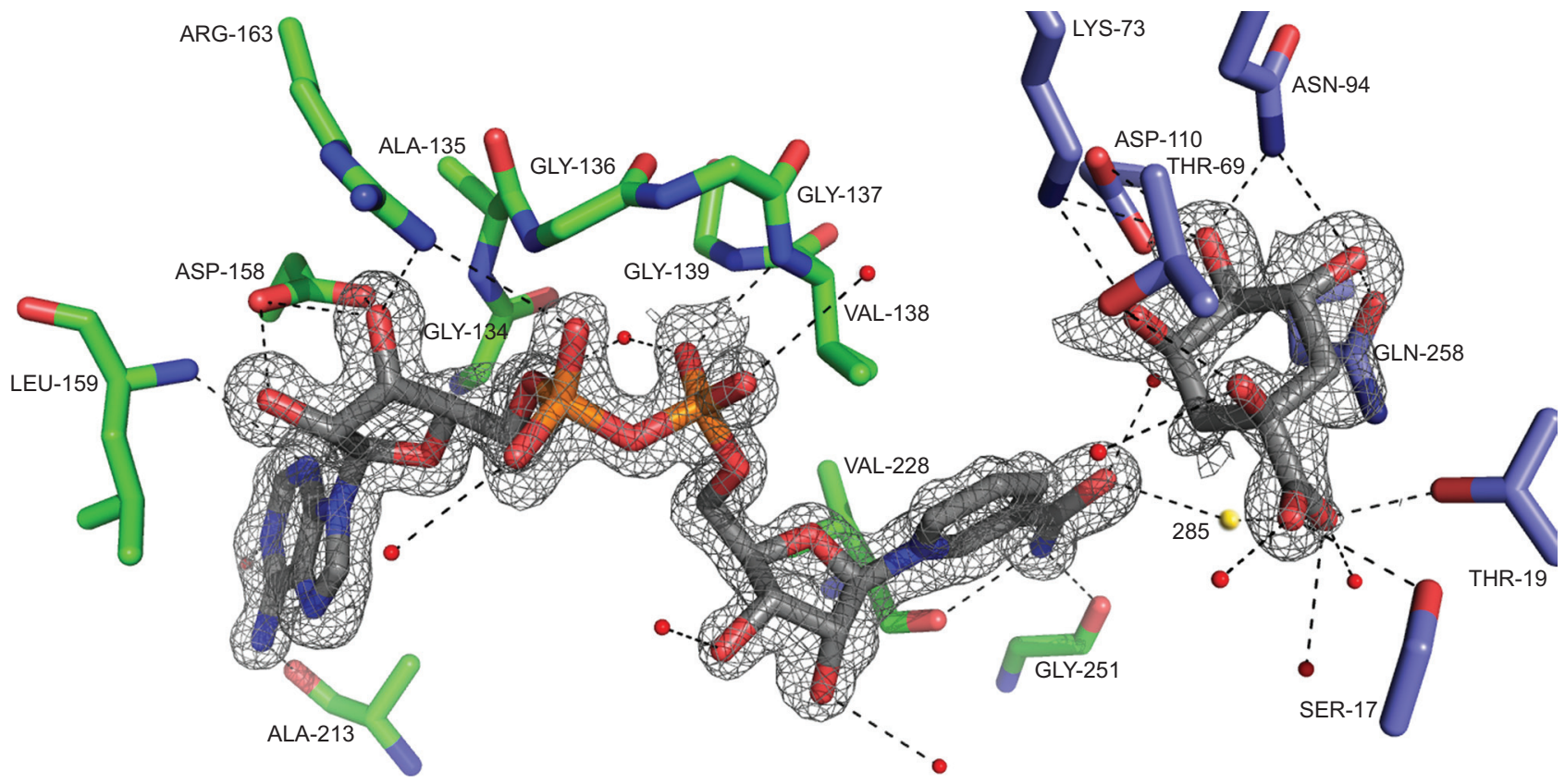

Figure 3 Active site of CglQSDH.

Residues involved in hydrogen bonds (dotted lines) and bound NADH and quinate are shown as stick models. Water molecules are depicted as red spheres. The putative catalytic water 285 is colored in yellow. The electron density around the ligands is contoured at $1 \sigma$.

A

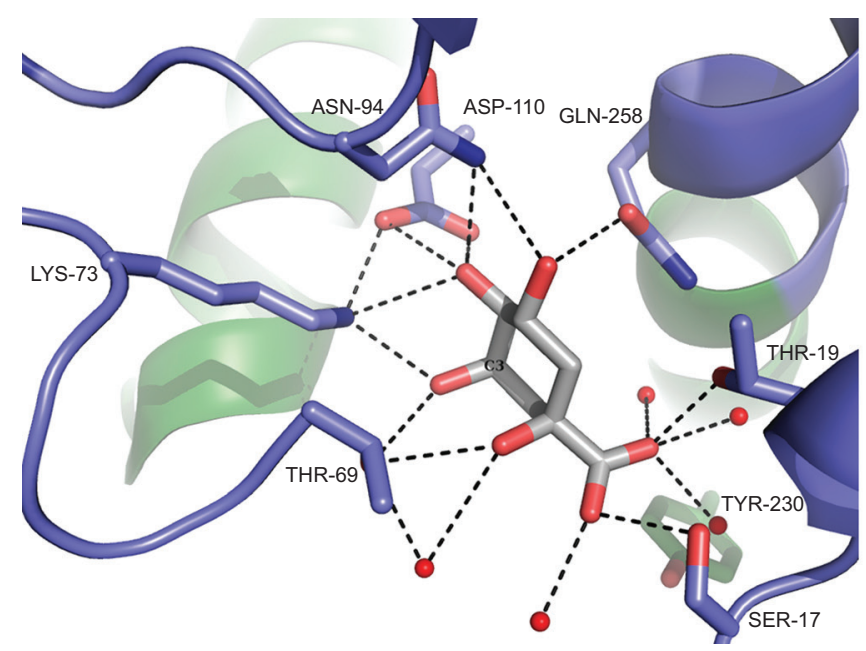

B

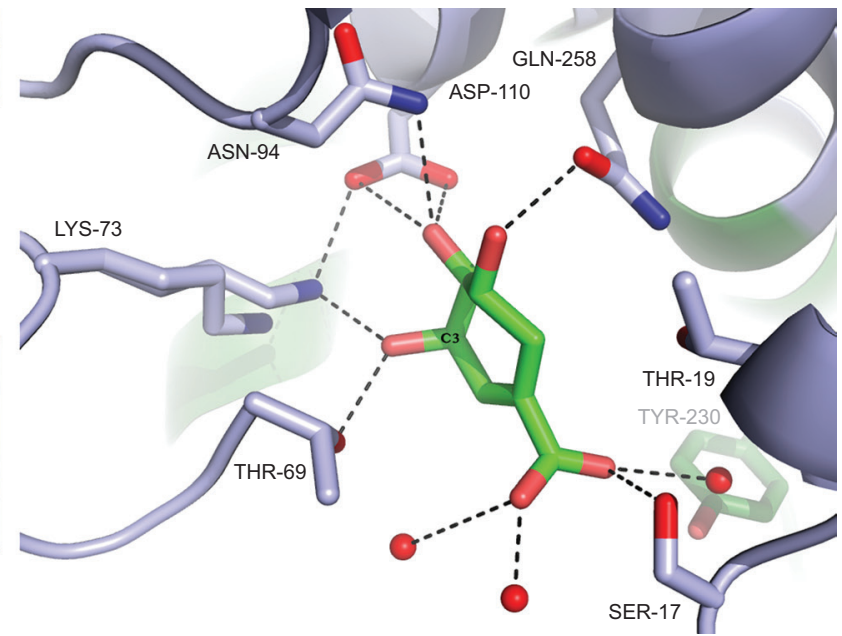

Figure 4 Substrate-binding pocket of CglQSDH.

Ternary complexes of $\mathrm{Cg} / \mathrm{QSDH}$ with bound quinate molecule in gray (A) or shikimate in green (B). Residues involved in hydrogen bonds (dotted lines) and bound ligand are shown as sticks. Water molecules are depicted as red spheres.

part of the side chains reveal an angled conformation as it occurs in apo-CglQSDH. The latter conformation makes hydrophobic interactions with the shikimate molecule impossible. Because of their different chemical properties, the shikimate molecule exists in a half-chair conformation, whereas the quinate molecule adopts a chair conformation. As a consequence, the shikimate molecule is fixed in a slightly different orientation compared with the bound quinate molecule. Hence, the distance of the $\mathrm{C} 4$ atom of the cofactor and the $\mathrm{C} 3$ atom of the substrate increases to $4.67 \AA$.

All residues involved in cofactor- and substrate-binding identified here are consistent with these of further reported structures (EcoYdiB, Benach et al., 2003; EcoAroE 
and EcoYdiB, Michel et al., 2003; MjaAroE, Padyana and Burley, 2003; HinAroE, Ye et al., 2003; AthSDH, Singh and Christendat, 2006, 2007; TthAroE, Bagautdinov and Kunishima, 2007; AaeAroE, Gan et al., 2007; SepAroE, Han et al., 2009; and PpuAel1, Peek et al., 2011). These highly conserved residues are highlighted in Figure S4 in the online supplement.

\section{Discussion}

\section{Substrate and cofactor specificity and discrimination}

All results of the structural analysis are in excellent agreement with the findings of the kinetic assays. The increased affinity of $C g l Q S D H$ to the substrate quinate compared with shikimate (as reflected by a lower $K_{\mathrm{m}}$ value, as shown in Table 1) is a consequence of a higher amount of hydrogen bonds (Table S3 in the online supplement). Considering the known structures of SDHs as AroE from T. thermophilus (Bagautdinov and Kunishima, 2007), AroE from A. aeolicus (Gan et al., 2007), or the SDH domain of A. thaliana (Singh and Christendat, 2006, 2007), they all possess at least 11 hydrogen bonds to the substrate molecule shikimate, comparable to the number of interactions between quinate and $\mathrm{CglQSDH}$. In contrast, there are only seven polar interactions present between the enzyme and the shikimate molecule resulting from the somewhat different conformation of the shikimate molecule (half-chair instead of chair conformation) as mentioned above. Additionally, the wellanchored hydroxy group at the $\mathrm{C} 1$ atom of the substrate quinate (Figure $4 \mathrm{~A}$ ) is absent in the shikimate molecule. Finally, the slightly different orientation of the shikimate molecule within the substrate-binding pocket compared with quinate does not allow optimal hydrogen bonds of all potential donors and acceptor groups. In summary, the details of the $C g l \mathrm{QSDH}$ active site allow an optimal exploitation of the entire hydrogen bonding potential in the case of quinate but not for shikimate.

Additionally, the lower affinity (and catalytic efficiency) regarding to shikimate may be caused by the appearance of an alternative conformation of the Lys73. One side-chain conformation exhibits a sprawled conformation as found for quinate binding, the other one reveals an angled conformation as it occurs in apo-CglQSDH, which leads to a loss of an important hydrogen bond, especially during catalysis.

Furthermore, we compared the substrate-binding residues of CglQSDH with those of AaeAroE, AthSDH, and TthAroE, which convert shikimate. We identified two major differences, which may be responsible for the improved quinate-binding mode in CglQSDH compared with the substrate shikimate. In most of the above-mentioned structures, there is a tyrosine residue involved in shikimate binding but which does not interact in $\mathrm{CglQSDH}$ (Tyr230, Figure 4). In AroE from S. epidermis, this residue (Tyr211) is likewise too far away from the substrate to form a hydrogen bond but it has been assigned to play a role in stabilizing the catalytic intermediate during catalysis (Han et al., 2009). In addition, the second serine, which forms a hydrogen bond to the carbonyl group of shikimate in the other SDH structures, is replaced by a threonine in CglQSDH (Thr19). Because the carbonyl group of shikimate bound in $\mathrm{CglQSDH}$ is twisted about $90^{\circ}$ compared with the situation of the aforesaid enzymes, Thr19 cannot take part in the polar interaction with the substrate shikimate.

Concerning the usage of the cofactors $\mathrm{NAD}(\mathrm{H})$ and $\operatorname{NADP}(\mathrm{H})$, several critical sequence motifs within the Rossmann fold were identified in the past. Mainly two regions are responsible for cofactor binding and discrimination. First, for all nucleotide-binding proteins, the glycine-rich loop is characteristic with the common sequence GxGxxG, in which the number of glycine residues changes (Rossmann et al., 1974). Second, within the next loop region of the nucleotide-binding $\beta \alpha \beta$ unit, the amino acid patterns of $\mathrm{NAD}(\mathrm{H})$ - and $\mathrm{NADP}(\mathrm{H})$-binding proteins are different: in general, enzymes using $\mathrm{NAD}^{+}$or FAD possess a well-conserved negatively charged amino acid at the $C$-terminus of the second $\beta$ strand of the nucleotide-binding $\beta \alpha \beta$ unit, mostly aspartate or glutamate. This residue interacts with the $2^{\prime}$-hydroxy group of the ribose. In contrast, in most of the NADP-binding proteins, this negatively charged residue is absent because the additional 2'-phospho group is located at this position (Wierenga et al., 1985, 1986; Baker et al., 1992). On the whole various NADP-dependent enzymes exhibit a charged amino acid (such as arginine) in the position of the 2'-phospho group, which stabilizes the cofactor molecule. Several representative members of the SDH family are strictly $\mathrm{NADP}(\mathrm{H})$ dependent and possess this above-mentioned sequence motif $\mathrm{NR}(\mathrm{D} / \mathrm{R} / \mathrm{T}) \mathrm{xx}(\mathrm{R} / \mathrm{K})$, as schematically shown in Figure 5. Examples are TthAroE, AaeAroE, AthSDH, and EcoAroE. EcoYdiB is assigned to use either $\mathrm{NAD}(\mathrm{H})$ as the only cofactor or both $\mathrm{NAD}(\mathrm{H})$ and NADP(H) (Benach et al., 2003; Michel et al., 2003). In the binary complexes with $\mathrm{NAD}(\mathrm{H})$, the latter protein exhibit a different hydrogen bonding pattern compared with the cofactor $\operatorname{NADP}(\mathrm{H})$ : the $2^{\prime}$-hydroxy group of the ribose interacts only with the conserved Asn155 and Asp158 of the cofactor-binding motif. 
A

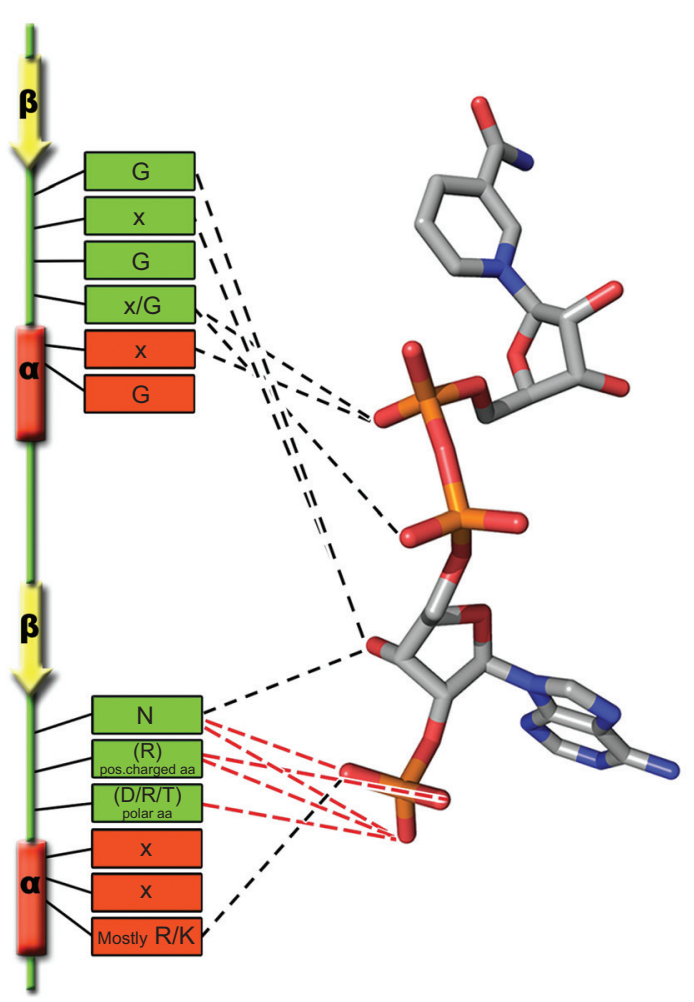

B

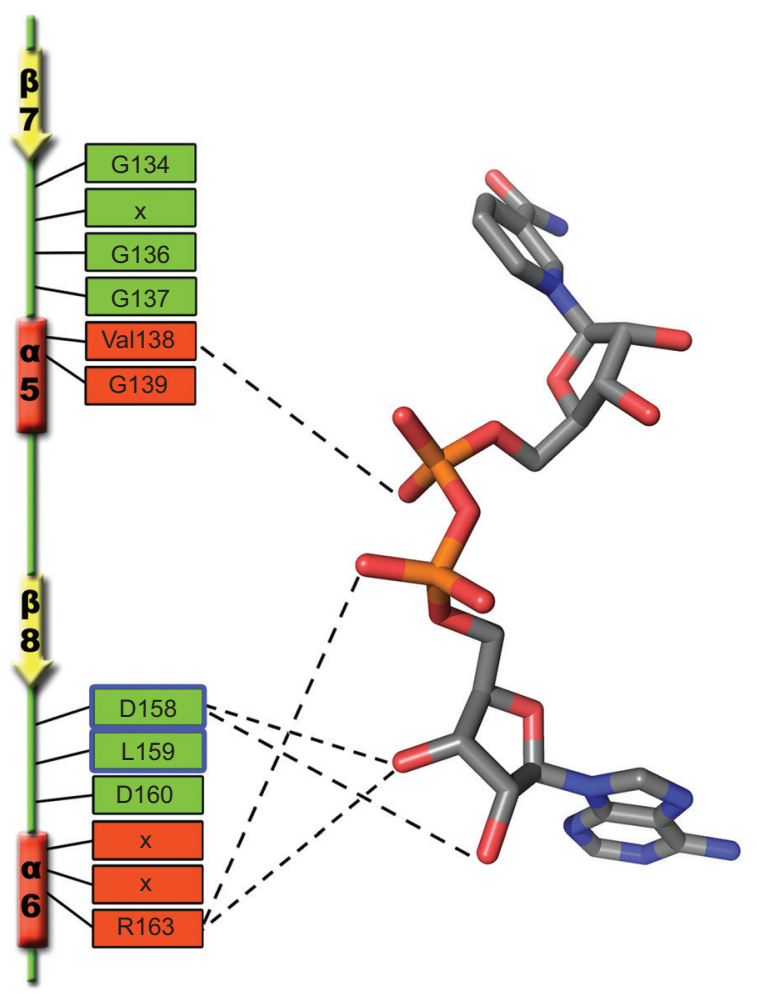

Figure 5 Typical sequence pattern of residues commonly involved in cofactor binding in NADP-dependent members of the SDH family (A) and the equivalent residues in the $\mathrm{NAD}^{+}$-dependent $\mathrm{Cg} / \mathrm{QSDH}$ (B).

(Upper left) Glycine-rich loop is shown. (Lower left) All residues responsible for cofactor binding and discrimination. Uncommon residues in the cofactor-binding region of $\mathrm{Cg} / \mathrm{QSDH}$ are highlighted with a blue frame. Hydrogen bonds are depicted as dashed lines, and those that are specific for $\mathrm{NADP}(\mathrm{H})$ binding are in red.

The CglQSDH described here offers a negatively charged residue (Asp158), forming a hydrogen bond to the $2^{\prime}$-hydroxy group of the ribose, followed by a neutral amino acid (Leu159), which is unable to interact with an additional phospho group as present in NADP(H). To our knowledge, CglQSDH and the recently described RifI2 enzyme, which has a higher activity using quinate and a strong preference for $\mathrm{NAD}^{+}$, are the only members of this enzyme class harbouring a neutral amino acid at that position, leucine in CglQSDH, proline in PpuRifl2 (Peek et al., 2013) (see multiple alignment in Figure $S 4$ in the online supplement).

All these findings are in good agreement with the results of the kinetic characterization of the YdiB2 protein (Singh et al., 2008), which has also a conserved aspartate instead of arginine as typical for NADP(H)-binding enzymes. YdiB2 is unable to use $\mathrm{NADP}(\mathrm{H})$ as a cofactor as well. Beyond that, the bulk side chain of Arg163 in CglQSDH constricts the cofactor-binding site, which would result in steric hindrance with the additional phospho group in a NADP(H) molecule.

The relative proximity between $\mathrm{CglQSDH}$ and PpuRifI2 (Peek et al., 2013) within the SDH/QDH family was further confirmed by a sequence- and structure-based comparison of CglQSDH with all SDH/QDH enzymes with structures available from the PDB using STRAP (Gille et al., 2003). After a first multiple sequence alignment, a nonredundant set of structures was created, which led to a collection of 20 unique SDH/QDH chains. Subsequently, these 20 structures were aligned against 3JYO (binary complex of CglQSDH with $\mathrm{NAD}^{+}$) as reference using the Aligner3D tool of STRAP, which combines structure and sequence information. In the resulting cladogram, the CglQSDH is localized separately from the four 'classical' subclasses of the SDH/QDH family but grouped together with the PpuRifI2 enzyme (Peek et al., 2013) (data not shown), which - as mentioned in the introduction - is the first member of a fifth subclass. However, a final classification for which we summarized kinetic and sequence data representative for all SDH/QDH subclasses (Table S5 in the online supplement) reveals even significant differences of CglQSDH to the novel 'Rifl' subclass (and to the four classical subclasses anyhow): (i) AroEs are strictly shikimate and $\mathrm{NADP}^{+}$dependent (highlighted in yellow); 
(ii) SDH-L is also strictly shikimate and $\mathrm{NADP}^{+}$dependent but with significantly lower catalytic efficiency (shaded in light blue); (iii) Ael1 is shikimate and $\mathrm{NADP}^{+}$dependent but exhibits residual activity with $\mathrm{NAD}^{+}$with regard to shikimate or residual activity with quinate with regard to $\mathrm{NADP}^{+}$(highlighted in terracotta) (it is not able to convert quinate with $\mathrm{NAD}^{+}$as a cofactor); (iv) YdiBs show dual substrate and cofactor specificity with slight preference for quinate and $\mathrm{NAD}^{+}$(shaded in blue); (v) RifI2 has dual substrate specificity with slight preference for quinate and residual activity with $\mathrm{NADP}^{+}$with regard to shikimate (shaded in light green) (it is not able to convert quinate with $\mathrm{NADP}^{+}$as a cofactor); and (vi) CglQSDH, which displays dual substrate specificity with preference for quinate but is strictly $\mathrm{NAD}^{+}$dependent (highlighted in orange).

Owing to all results of the kinetic and structural analyses as well as of the sequence analyses, we conclude that $C g l \mathrm{QSDH}$ is strictly $\mathrm{NAD}(\mathrm{H})$ specific and cannot bind $\operatorname{NADP}(\mathrm{H})$. Based on structure and sequence properties, we could identify amino acids patterns that define cofactor specificity and discrimination. By comparing both ternary complexes, we were able to specify the residues involved in hydrophilic interactions with the substrates quinate or shikimate, which allow to explain the different substrate affinities and catalytic efficiencies with these molecules.

\section{Materials and methods}

\section{Chemicals}

Ni-NTA was obtained from Qiagen (Hilden, Germany). Shikimic and quinic acid as well as $\mathrm{NAD}(\mathrm{P})^{+}$and $\mathrm{NAD}(\mathrm{P}) \mathrm{H}$ were purchased from Sigma (Sigma-Aldrich Chemie Gmbh, Munich, Germany). All other chemicals were ordered from Merck (Merck KGaA, Darmstadt, Germany).

\section{Cloning, expression, and purification}

The gene encoding the QSDH (cgl0424) was amplified by polymerase chain reaction from $C$. glutamicum strain ATCC13032 genomic DNA and cloned into the pNHIS vector as described elsewhere (Chatterjee et al., 2005). Competent $E$. coli strain BL21 cells were transformed with the pNHIS-QSDH vector. Bacterial LB culture $(250 \mathrm{ml})$ supplemented with $150 \mathrm{mg} / \mathrm{l}$ ampicillin was grown at $37^{\circ} \mathrm{C}$ to an optical density of $\sim 0.6$ at $600 \mathrm{~nm}$ on an orbital shaker (New Brunswick Scientific Co. Ing., NJ, USA). The culture was then induced by adding isopropyl$\beta$-D-thiogalactopyranoside to a final concentration of $1 \mathrm{mM}$. Overexpression was conducted by vigorous shaking at $37^{\circ} \mathrm{C}$ for $4 \mathrm{~h}$. Cells were harvested by centrifugation and frozen at $-20^{\circ} \mathrm{C}$. Pellets from $500 \mathrm{ml}$ cell culture were resuspended in $40 \mathrm{ml}$ lysis buffer $(50 \mathrm{~mm}$
$\mathrm{NaH}_{2} \mathrm{PO}_{4} / \mathrm{Na}_{2} \mathrm{HPO}_{4}$, $\mathrm{pH} 8.0,300 \mathrm{~mm} \mathrm{NaCl}, 10 \mathrm{~mm}$ imidazole) and then disrupted by sonication at $4^{\circ} \mathrm{C}$ (Bandelin, Berlin, Germany). Insoluble cellular material was removed by centrifugation. QSDH was purified from other contaminating proteins using nickel-nitrilotriacetic acid affinity chromatography. Elution buffer contained $50 \mathrm{~mm} \mathrm{NaH}_{2}$ $\mathrm{PO}_{4} / \mathrm{Na}_{2} \mathrm{HPO}_{4}, \mathrm{pH}$ 8.0, $300 \mathrm{~mm} \mathrm{NaCl}, 250 \mathrm{~mm}$ imidazole. Protein fractions were analyzed via SDS-PAGE. Purified QSDH was concentrated and quantified by the method of Bradford.

\section{Enzyme kinetics}

The enzymatic activity of QSDH was assayed by monitoring the reduction of $\mathrm{NAD}^{+}$at $340 \mathrm{~nm}$ and $30^{\circ} \mathrm{C}$ in the presence of either quinate or shikimate using the molar extinction coefficient $\varepsilon_{\mathrm{NAD}(\mathrm{P}) \mathrm{H}}=6220 \mathrm{M}^{-1} \mathrm{~cm}^{-1}$. For the determination of enzyme $\mathrm{pH}$ optimum, two different buffer systems were applied (Tris- $\mathrm{HCl}$ for $\mathrm{pH}$ 7.0-9.5 and sodium carbonate for $\mathrm{pH}$ 8.5-11.5). Substrate concentrations were $8 \mathrm{~mm}$ for quinate, $50 \mathrm{~mm}$ for shikimate, and $0.5 \mathrm{~mm}$ for $\mathrm{NAD}^{+}$. The $K_{\mathrm{m}}$ and $V_{\max }$ values were determined by varying the concentrations of quinate, shikimate, or $\mathrm{NAD}^{+}$while keeping the other substrate at saturation. Quinate was varied from 0.25 to $32 \mathrm{~mm}$, shikimate from 25 to $60 \mathrm{~mm}$, NAD ${ }^{+}$from 15.6 to $10 \mathrm{~mm}$. All measurements were done at $\mathrm{pH}$ optimum ( $\mathrm{pH}$ 9.0/9.5

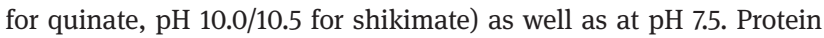
concentrations were $5 \mathrm{~nm}$ for quinate and shikimate at $\mathrm{pH}$ optimum and $12.5 \mathrm{~nm}$ for quinate at $\mathrm{pH} 7.5$ or $25 \mathrm{~nm}$ for shikimate, respectively. All measurements were carried out as triple assay with two different batches at least. The ability of QSDH to use $\mathrm{NADP}^{+}$as cofactor was verified by replacing $\mathrm{NAD}^{+}$with $\mathrm{NADP}^{+}$. The $K_{\mathrm{m}}$ and $k_{\text {cat }}$ values were calculated using the computer program of GraFit (Erithacus Software Limited, Surrey, UK). To ensure that the N-terminal his-tag does not influence enzymatic activity, it was cleaved using the site-specific AcTEV protease (Invitrogen, Life Technologies, Carlsbad, CA, USA). The result of cleavage was controlled by mass spectrometry.

\section{Crystallization and X-ray diffraction data collection}

For crystallization, the buffer of protein solution was changed by filtration (50 mm Tris- $\mathrm{HCl}, \mathrm{pH} 7.5,500 \mathrm{~mm} \mathrm{NaCl,} 20 \%$ v/v glycerol). Crystals of QSDH in complex with $\mathrm{NAD}^{+}$or as ternary complex with $\mathrm{NADH}$ and quinate or shikimate were grown using the vapor diffusion sitting-drop method at $20^{\circ} \mathrm{C}$. The crystallization condition contained $2 \mu \mathrm{l}$ of protein solution $(-10 \mathrm{mg} / \mathrm{ml}$ containing $1 \mathrm{~mm}$ of $\mathrm{NAD}^{+} / \mathrm{NADH}$, and for the ternary complexes, additionally $35 \mathrm{~mm}$ of either quinate or shikimate, preincubated for $1 \mathrm{~h}$ at $4^{\circ} \mathrm{C}$ ) and $2 \mu \mathrm{l}$ of precipitant. Crystallization solution of the QSDH-NAD ${ }^{+}$crystals contained 1.6 $\mathrm{M}$ trisodium citrate, $\mathrm{pH} 6.5-6.9,25-62 \mathrm{~mm} \mathrm{CoCl}_{2}$. Crystals of the QSDH-quinate-NADH and QSDH-shikimate-NADH grown in $24 \% \mathrm{w} / \mathrm{v}$ polyethylene glycol 6000, 360-400 mM CaCl, $100 \mathrm{~mm}$ Tris-HCl, pH 8.0-9.5, were soaked in a cryoprotectant containing $30 \% \mathrm{w} / \mathrm{v}$ polyethylene glycol $6000,25 \% \mathrm{v} / \mathrm{v}$ glycerol, $100 \mathrm{~mm}$ Tris$\mathrm{HCl}, \mathrm{pH} 8.5$, for $1 \mathrm{~h}$. The resulting crystals of QSDH-NAD ${ }^{+}$diffracted to $1.0 \AA$, crystals of QSDH-quinate-NADH and QSDH-shikimateNADH to $1.16 \AA$, respectively. The diffraction data at high and lower resolutions were collected from a single crystal at $100 \mathrm{~K}$ in a nitrogen stream (EMBL Outstation; DESY, Hamburg, Germany). All crystals belong to the space group $\mathrm{C} 2$ with similar unit cell parameters 
( $a=121 \AA, b=63 \AA, c=36 \AA, \beta=94^{\circ}$ ) and one monomer per asymmetric unit. Reflection data were indexed, integrated, and scaled using DENZO and SCALEPACK (Otwinowski and Minor, 1997).

\section{Structure determination, model building, and refinement}

The crystal structures of all complexes were determined by the method of molecular replacement. The coordinates of the isomorphous structure of the apo-CglQSDH, PDB code 2NLO (Schoepe et al., 2008), were used as template for model building and refinement. Water molecules were fitted in using the program ARP/wARP (Perrakis et al., 2001). Coordinates of the substrates quinate, shikimate, and $\mathrm{NAD}(\mathrm{H})$ were isolated from a protein complex from PDB and converted in REFMAC format using the PRODRG2 server. The fitting of the substrates was carried out manually. For refinement, we performed iterative cycles of manual modulation with the program 0 (Jones et al., 1991) followed by molecular refinement using REFMAC (Collaborative Computational Project, 1994).

The final models have good stereochemistry, as determined by the programs PROCHECK and REFMAC as well as by the validation server of the PDB. RMSD values were calculated using the program Superpose from the ccp4 suite (Krissinel and Henrick, 2004). The figures were generated using PyMOL (DeLano Scientific LLC, San Carlos, CA, USA).

\section{Similarity analysis}

For multiple protein sequence alignment, the CLUSTALW2 service from the EMBL-EBI website (http://www.ebi.ac.uk/Tools/ msa/clustalw2/) was used. For a sequence- and structure-based comparison of $\mathrm{CglQSDH}$ with all SDH/QDH enzymes with structures available from the PDB, we used STRAP (Gille et al., 2003) as a master tool. The CglQSDH-NAD complex structure (PDB 3JYO) was loaded into the STRAP environment. All proteins with similar sequences and published 3D structures were identified, from which chain A was loaded as well. After a first multiple sequence alignment using ClustalW (out of STRAP), a nonredundant set of structures was created; a sequence identity cutoff of $98 \%$ led to a set of 20 unique SDH/ QDH chains (out of 41 SDH/QDH PDB files on the whole).

Subsequently, these 20 structures were aligned against 3 JYO as reference using the Aligner3D tool of STRAP, which combines structure and sequence information.

Acknowledgments: We thank Dr. Jan Schoepe for the kind allocation of the CglQSDH-pNHIS vector and Sabine Lohmer for excellent technical assistance. We acknowledge the European Synchrotron Radiation Facility for provision of synchrotron radiation facilities for the initial screening of the crystals and the staff of the EMBL Outstation, DESY Hamburg, Germany, for their friendly help during data collection at the X12 and BW7B beamlines. We thank Dr. Sander Smits for productive discussion and critical reading of the manuscript. We gratefully acknowledge support from the International NRW Research School BioStruct, granted by the Ministry of Innovation, Science and Research of the State North Rhine-Westphalia, the Heinrich Heine University of Düsseldorf, and the Entrepreneur Foundation at the Heinrich-Heine-University of Düsseldorf. Astrid Höppner also thanks the 'Fit For Excellence' Fund of the Heinrich Heine University for subsequent financing.

Received May 4, 2013; accepted August 5, 2013; previously published online August 7, 2013

\section{References}

Bagautdinov, B. and Kunishima, N. (2007). Crystal structures of shikimate dehydrogenase AroE from Thermus thermophilus HB8 and its cofactor and substrate complexes: insights into the enzymatic mechanism. J. Mol. Biol. 373, 424-438.

Baker, P.J., Britton, K.L., Rice, D.W., Rob, A., and Stillman, T.J. (1992). Structural consequences of sequence patterns in the fingerprint region of the nucleotide binding fold. J. Mol. Biol. 228, 662-671.

Benach, J., Lee, I., Edstrom, W., Kuzin, A.P., Chiang, Y., Acton, T.B., Montelione, G.T., and Hunt, J.F. (2003). The 2.3-A crystal structure of the shikimate 5-dehydrogenase orthologue YdiB from Escherichia coli suggests a novel catalytic environment for an NAD-dependent dehydrogenase. J. Biol. Chem. 278, 19176-19182.

Bruce, N.C. and Cain, R.B. (1990). Hydroaromatic metabolism in Rhodococcus rhodochrous: purification and characterisation of its NAD-dependent quinate dehydrogenase. Arch. Microbiol. 154, 179-186.
Cain, R.B. (1972). The identity of shikimate dehydrogenase and quinate dehydrogenase in Aspergillus niger. Biochem. J. 127, $15 \mathrm{P}$.

Carpenter, E.P., Hawkins, A.R., Frost, J.W., and Brown, K.A. (1998). Structure of dehydroquinate synthase reveals an active site capable of multistep catalysis. Nature 394, 299-302.

Charles, I.G., Keyte, J.W., Brammar, W.J., and Hawkins, A.R. (1985). Nucleotide sequence encoding the biosynthetic dehydroquinase function of the penta-functional arom locus of Aspergillus nidulans. Nucl. Acids Res. 13, 8119-8128.

Chatterjee, S., Schoepe, J., Lohmer, S., and Schomburg, D. (2005). High level expression and single-step purification of hexahistidine-tagged L-2-hydroxyisocaproate dehydrogenase making use of a versatile expression vector set. Protein Exp. Purif. 39, 137-143.

Chaudhuri, S. and Coggins, J.R. (1985). The purification of shikimate dehydrogenase from Escherichia coli. Biochem. J. 226, 217-223. 
Coggins, J.R., Abell, C., Evans, L.B., Frederickson, M., Robinson, D.A., Roszak, A.W., and Lapthorn, A.P. (2003). Experiences with the shikimate-pathway enzymes as targets for rational drug design. Biochem. Soc. Trans. 31, 548-552.

Collaborative Computational Project (1994). The CCP4 suite: programs for protein crystallography. Acta Crystallogr. D Biol. Crystallogr. 50, 760-763.

Davies, G.M., Barrett-Bee, K.J., Jude, D.A., Lehan, M., Nichols, W.W., Pinder, P.E., Thain, J.L., Watkins, W.J., and Wilson, R.G. (1994). (6S)-6-fluoroshikimic acid, an antibacterial agent acting on the aromatic biosynthetic pathway. Antimicrob. Agents Chemother. 38, 403-406.

Elsemore, D.A. and Ornston, L.N. (1994). The pca-pob supraoperonic cluster of Acinetobacter calcoaceticus contains quiA, the structural gene for quinate-shikimate dehydrogenase. J. Bacteriol. 176, 7659-7666.

Gan, J., Wu, Y., Prabakaran, P., Gu, Y., Li, Y., Andrykovitch, M., Liu, H., Gong, Y., Yan, H., and Ji, X. (2007). Structural and biochemical analyses of shikimate dehydrogenase AroE from Aquifex aeolicus: implications for the catalytic mechanism. Biochemistry 46, 9513-9522.

Gille, C., Lorenzen, S., Michalsky, E., and Frommel, C. (2003). KISS for STRAP: user extensions for a protein alignment editor. Bioinformatics 19, 2489-2491.

Gourley, D.G., Shrive, A.K., Polikarpov, I., Krell, T., Coggins, J.R., Hawkins, A.R., Isaacs, N.W., and Sawyer, L. (1999). The two types of 3-dehydroquinase have distinct structures but catalyze the same overall reaction. Nat. Struct. Biol. 6, 521-525.

Grund, E. and Kutzner, H.J. (1998). Utilization of quinate and p-hydroxybenzoate by actinomycetes: key enzymes and taxonomic relevance. J. Basic Microbiol. 38, 241-255.

Han, C., Hu, T., Wu, D., Qu, S., Zhou, J., Ding, J., Shen, X., Qu, D., and Jiang, H. (2009). X-ray crystallographic and enzymatic analyses of shikimate dehydrogenase from Staphylococcus epidermidis. FEBS J. 276, 1125-1139.

Harwood, C.S. and Parales, R.E. (1996). The beta-ketoadipate pathway and the biology of self-identity. Annu. Rev. Microbiol. 50, 553-590.

Herrmann, K.M. (1995). The shikimate pathway: early steps in the biosynthesis of aromatic compounds. Plant Cell 7, 907-919.

Herrmann, K.M. and Weaver, L.M. (1999). The shikimate pathway. Annu. Rev. Plant Physiol. Plant Mol. Biol. 50, 473-503.

Ikeda, M. and Katsumata, R. (1992). Metabolic engineering to produce tyrosine or phenylalanine in a tryptophan-producing Corynebacterium glutamicum strain. Appl. Environ. Microbiol. 58, 781-785.

Jones, T.A., Zou, J.Y., Cowan, S.W., and Kjeldgaard, M. (1991). Improved methods for building protein models in electron density maps and the location of errors in these models. Acta Crystallogr. A Found. Crystallogr. 47, 110-119.

Kang, X. and Scheibe, R. (1993). Purification and characterization of the quinate:oxidoreductase from Phaseolus mungo sprouts. Phytochemistry 33, 769-773.

Krissinel, E. and Henrick, K. (2004). Secondary-structure matching (SSM), a new tool for fast protein structure alignment in three dimensions. Acta Crystallogr. D Biol. Crystallogr. 60, $2256-2268$.
Kubota, T., Tanaka, Y., Hiraga, K., Inui, M., and Yukawa, H. (2013). Characterization of shikimate dehydrogenase homologues of Corynebacterium glutamicum. Appl. Microbiol. Biotechnol. Jan 2013; DOI 10.1007/s00253-012-4659-y). [Epub ahead of print].

Lindner, H.A., Nadeau, G., Matte, A., Michel, G., Menard, R., and Cygler, M. (2005). Site-directed mutagenesis of the active site region in the quinate/shikimate 5 -dehydrogenase $\mathrm{YdiB}$ of Escherichia coli. J. Biol. Chem. 280, 7162-7169.

Maclean, J. and Ali, S. (2003). The structure of chorismate synthase reveals a novel flavin binding site fundamental to a unique chemical reaction. Structure 11, 1499-1511.

Michel, G., Roszak, A.W., Sauve, V., Maclean, J., Matte, A., Coggins, J.R., Cygler, M., and Lapthorn, A.J. (2003). Structures of shikimate dehydrogenase AroE and its Paralog YdiB. A common structural framework for different activities. J. Biol. Chem. 278, 19463-19472.

Mitsuhashi, S. and Davis, B.D. (1954). Aromatic biosynthesis. XIII. Conversion of quinic acid to 5 -dehydroquinic acid by quinic dehydrogenase. Biochim. Biophys. Acta 15, 268-280.

Otwinowski, Z. and Minor, W. (1997). Processing of X-ray diffraction data collected in oscillation mode. Methods Enzymol. 276, 307-326.

Padyana, A.K. and Burley, S.K. (2003). Crystal structure of shikimate 5-dehydrogenase (SDH) bound to NADP: insights into function and evolution. Structure 11, 1005-1013.

Peek, J., Lee, J., Hu, S., Senisterra, G., and Christendat, D. (2011). Structural and mechanistic analysis of a novel class of shikimate dehydrogenases: evidence for a conserved catalytic mechanism in the shikimate dehydrogenase family. Biochemistry 50, 8616-8627.

Peek, J., Garcia, C., Lee, J., and Christendat, D. (2013). Insights into the function of Rifl2: structural and biochemical investigation of a new shikimate dehydrogenase family protein. Biochim. Biophys. Acta 1834, 516-523.

Perrakis, A., Harkiolaki, M., Wilson, K.S., and Lamzin, V.S. (2001). ARP/wARP and molecular replacement. Acta Crystallogr. D Biol. Crystallogr. 57, 1445-1450.

Reinert, W.R., Patel, V.B., and Giles, N.H. (1981). Genetic regulation of the qa gene cluster of Neurospora crassa: induction of qa messenger ribonucleic acid and dependency on qa-1 function. Mol. Cell. Biol. 1, 829-835.

Roberts, F., Roberts, C.W., Johnson, J.J., Kyle, D.E., Krell, T., Coggins, J.R., Coombs, G.H., Milhous, W.K., Tzipori, S., Ferguson, D.J., et al. (1998). Evidence for the shikimate pathway in apicomplexan parasites. Nature 393, 801-805.

Rodrigues, V.S., Jr., Breda, A., Santos, D.S., and Basso, L.A. (2009). The conserved Lysine69 residue plays a catalytic role in Mycobacterium tuberculosis shikimate dehydrogenase. BMC Res. Notes 2, 227.

Rossmann, M.G., Moras, D., and Olsen, K.W. (1974). Chemical and biological evolution of nucleotide-binding protein. Nature 250 , 194-199.

Roszak, A.W., Robinson, D.A., Krell, T., Hunter, I.S., Fredrickson, M., Abell, C., Coggins, J.R., and Lapthorn, A.J. (2002). The structure and mechanism of the type II dehydroquinase from Streptomyces coelicolor. Structure 10, 493-503.

Sahm, H., Eggeling, L., and de Graaf, A.A. (2000). Pathway analysis and metabolic engineering in Corynebacterium glutamicum. Biol. Chem. 381, 899-910. 
Schoepe, J., Niefind, K., Chatterjee, S., and Schomburg, D. (2006). Cloning, expression, purification and preliminary crystallographic characterization of a shikimate dehydrogenase from Corynebacterium glutamicum. Acta Crystallogr. F Struct. Biol. Cryst. Commun. 62, 635-637.

Schoepe, J., Niefind, K., and Schomburg, D. (2008). 1.6 angstroms structure of an NAD+-dependent quinate dehydrogenase from Corynebacterium glutamicum. Acta Crystallogr. D Biol. Crystallogr. D64, 803-809.

Schomburg, I., Chang, A., Placzek, S., Sohngen, C., Rother, M., Lang, M., Munaretto, C., Ulas, S., Stelzer, M., Grote, A., et al. (2013). BRENDA in 2013: integrated reactions, kinetic data, enzyme function data, improved disease classification: new options and contents in BRENDA. Nucl. Acids Res. 41, D764-772.

Shumilin, I.A., Kretsinger, R.H., and Bauerle, R.H. (1999). Crystal structure of phenylalanine-regulated 3-deoxy-D-arabinoheptulosonate-7-phosphate synthase from Escherichia coli. Structure 7, 865-875.

Singh, S.A. and Christendat, D. (2006). Structure of Arabidopsis dehydroquinate dehydratase-shikimate dehydrogenase and implications for metabolic channeling in the shikimate pathway. Biochemistry 45, 7787-7796.

Singh, S.A. and Christendat, D. (2007). The DHQ-dehydroshikimateSDH-shikimate-NADP(H) complex: insights into metabolite transfer in the shikimate pathway. Crystal Growth Des. 7, 2153-2160.

Singh, S., Korolev, S., Koroleva, O., Zarembinski, T., Collart, F., Joachimiak, A., and Christendat, D. (2005). Crystal structure of a novel shikimate dehydrogenase from Haemophilus influenzae. J. Biol. Chem. 280, 17101-17108.
Singh, S., Stavrinides, J., Christendat, D., and Guttman, D.S. (2008). A phylogenomic analysis of the shikimate dehydrogenases reveals broadscale functional diversification and identifies one functionally distinct subclass. Mol. Biol. Evol. 25, 2221-2232.

Stallings, W.C., Abdel-Meguid, S.S., Lim, L.W., Shieh, H.S., Dayringer, H.E., Leimgruber, N.K., Stegeman, R.A., Anderson, K.S., Sikorski, J.A., Padgette, S.R., et al. (1991). Structure and topological symmetry of the glyphosate target 5-enolpyruvylshikimate-3-phosphate synthase: a distinctive protein fold. Proc. Natl. Acad. Sci. USA 88, 5046-5050.

Teramoto, H., Inui, M., and Yukawa, H. (2009). Regulation of expression of genes involved in quinate and shikimate utilization in Corynebacterium glutamicum. Appl. Environ. Microbiol. 75, 3461-3468.

Whiting, G.C. and Coggins, R.A. (1974). A new nicotinamide-adenine dinucleotide-dependent hydroaromatic dehydrogenase of Lactobacillus plantarum and its role in formation of (minus) t-3,t-4-dihydroxycyclohexane-c-1-carboxylate. Biochem. J. 141, 35-42.

Wierenga, R.K., De Maeyer, M.C.H., and Hol, W.G.J. (1985). Interaction of pyrophosphate moieties with $\alpha$-helixes in dinucleotide binding proteins. Biochemistry 24, 1346-1357.

Wierenga, R.K., Terpstra, P., and Hol, W.G. (1986). Prediction of the occurrence of the ADP-binding $\beta \alpha \beta$-fold in proteins, using an amino acid sequence fingerprint. J. Mol. Biol. 187, 101-107.

Yaniv, H. and Gilvarg, C. (1955). Aromatic biosynthesis XIV. 5-dehyroshikimic reductase. J. Biol. Chem. 213, 787-795.

Ye, S., Von Delft, F., Brooun, A., Knuth, M.W., Swanson, R.V., and McRee, D.E. (2003). The crystal structure of shikimate dehydrogenase (AroE) reveals a unique NADPH binding mode. J. Bacteriol. 185, 4144-4151. 